\title{
Uso do Sistema Manométrico Miniaturizado Pressure-Wire em Cardiopatias Estruturais Congênitas e Adquiridas
}

\author{
Gustavo Caires Novaes, Jorge Luis Haddad, Daniel Conterno Lemos, Andre Vannucchi Badran, \\ Rafael Brolio Pavão, Igor Matos Lago, Moyses de Oliveira Lima-Filho, \\ Geraldo Luiz de Figueiredo, J. Antonio Marin-Neto
}

\section{RESUMO}

Introdução: Historicamente, o registro de pressões intravasculares contribuiu para o desenvolvimento da cardiologia intervencionista. Embora atualmente novos recursos de imagem sejam preponderantes, a medida acurada de pressões intravasculares ainda é essencial em muitos contextos diagnósticos e de aferição de métodos terapêuticos intervencionistas. Descrevemos a utilização do sistema manométrico miniaturizado (pressure-wire) para obtenção de curvas pressóricas em cardiopatias estruturais congênitas e adquiridas. Métodos: Foram utilizados os sistemas RADI Analyzer ${ }^{\circledR}$ Xpress (St. Jude Medical Inc., St. Paul, Minnesota, Estados Unidos) e PressureWire ${ }^{\circledR}$ Certus durante procedimentos com cateteres diagnósticos $5 \mathrm{~F}$ realizados sob anestesia geral e suporte ventilatório em crianças. Traçados manométricos foram obtidos simultaneamente com o pressure-wire e o cateter $5 \mathrm{~F}$ em pacientes para os quais era imperativa a decisão terapêutica com base em análise das pressões intravasculares e nos quais não era possível obtê-las acuradamente pelos métodos convencionais. Resultados: O pressure-wire foi utilizado para obtenção de pressões intravasculares pulmonares em pacientes com colaterais sistêmico-pulmonares, com estenoses angiográficas ou não, e com variadas cardiopatias estruturais, na avaliação de estenoses de ramos pulmonares, na localização de estenoses de condutos cirúrgicos (por exemplo: após cirurgia de Rastelli), shunts cirúrgicos (como de Blalock-Taussig) e na avaliação pré-operatória de derivações cavo-pulmonares. O procedimento foi realizado com segurança, tendo sido obtidos traçados manométricos com qualidade adequada. Conclusões: O sistema manométrico miniaturizado está consagrado no cenário da cardiologia intervencionista, enquanto modalidade diagnóstica complementar para avaliação funcional de lesões coronárias. Esse sistema também constitui método diagnóstico complementar em diversas cardiopatias estruturais, congênitas e adquiridas.

DESCRITORES: Cardiopatias congênitas. Cateterismo cardíaco. Hemodinâmica.

\section{ABSTRACT}

\author{
Use of the Miniature Pressure-Wire \\ Manometric System in Congenital and \\ Acquired Structural Heart Diseases
}

Background: Historically, intravascular pressure recording has contributed to the development of interventional cardiology. Although new imaging resources have gained much attention, accurate intravascular pressure measurement remains essential for the diagnosis and evaluation of interventional therapy methods. We describe the use of the miniature manometric system (pressure-wire) to obtain pressure curves in congenital and acquired structural heart diseases. Methods: The Radi Analyzer ${ }^{\circledR}$ Xpress (St. Jude Medical Inc., St. Paul, MN, USA) and PressureWire ${ }^{\circledR}$ Certus systems were used in procedures with $5 \mathrm{~F}$ catheters under general anesthesia and ventilatory support in children. Manometric tracings were obtained simultaneously from pressure-wire and the $5 \mathrm{~F}$ catheter in patients whose therapy strategies were dependent on the analysis of intravascular pressures, and in whom was not possible to obtain them accurately by conventional methods. Results: Pressure-wire was used to obtain pulmonary intravascular pressures in patients with systemic-pulmonary collaterals with or without angiographically detected stenosis, and with different structural heart diseases, in the evaluation of pulmonary branch stenosis, in the localization of surgical conduit stenosis (e.g. after Rastelli surgery), surgical shunts (such as Blalock-Taussig) and in the preoperative evaluation of cavopulmonary shunts. The procedure was performed safely, and manometric tracings were obtained with an adequate quality. Conclusions: The miniature manometric system is well accepted as a complementary diagnostic modality for the functional assessment of coronary lesions in interventional cardiology. It is also a complementary diagnostic method in different structural, congenital and acquired heart diseases.

DESCRIPTORS: Heart defects, congenital. Cardiac catheterization. Hemodynamics.
Hospital das Clínicas, Faculdade de Medicina de Ribeirão Preto, Universidade de São Paulo, São Paulo, SP, Brasil.
Correspondência: Gustavo Caires Novaes. Avenida Bandeirantes, 3.900 CEP 14848-900 - Ribeirão Preto, SP, Brasil

E-mail: gustavonovaes@hotmail.com.

Recebido em: 3/1/2014 • Aceito em: 25/2/2014 
0 registro de curvas pressóricas que explicassem as manifestações físicas do funcionamento do sistema cardiovascular foi um dos propulsores essenciais para o desenvolvimento da hemodinâmica moderna, como aplicada ao estudo da fisiologia e da fisiopatologia humanas. Os estudos pioneiros de Claude Bernard, citados por Buzzi, em 1959,1 levaram à cateterização de câmaras cardíacas em 1847, com o registro pressórico do ventrículo direito (VD) em animais de experimentação.

Pouco menos de um século depois, seguindo-se à autoexperiência de Forssman, em 1929, os trabalhos subsequentes de André Cournand e de vários outros pesquisadores impulsionaram decisivamente o conhecimento da fisiopatologia de doenças congênitas e adquiridas, por meio de registros pressóricos e manometria das câmaras cardíacas direitas, e da circulação pulmonar. Paralelamente, métodos de oximetria e de análise de curvas de diluição de diversos indicadores foram desenvolvidos para medida do débito cardíaco, e detecção e quantificação de derivações ou comunicações (shunts) entre as circulações sistêmica e pulmonar. Pela associação dos dados manométricos a esses métodos, possibilitou-se a mensuração de resistências vasculares e ao nível das valvas cardíacas, em condição normal e na presença de defeitos cardiovasculares estruturais, congênitos e adquiridos..$^{2,3}$

Em décadas mais recentes, grande interesse tem sido dado à angiografia de contraste radiológico e a outros métodos de imageamento cardiovascular durante o cateterismo cardíaco, como a ultrassonografia intracoronária e a tomografia de coerência óptica, por exemplo. Infelizmente, o aproveitamento prático de tais avanços tecnológicos tem ocorrido simultaneamente a certo desinteresse dos Cardiologistas Intervencionistas pelos métodos clássicos de Hemodinâmica - aí incluídos os registros pressóricos, as mensurações de fluxo sanguíneo e os cálculos de resistências vasculares e de orifícios valvares.

Em se tratando de doenças cardíacas congênitas, a determinação de pressões intravasculares e em câmaras cardíacas tem especial importância, uma vez que são definidoras da modalidade de tratamento a seguir, por influenciarem na escolha da técnica cirúrgica, ocasionalmente contraindicando ou condicionando a terapêutica paliativa. No cenário de algumas dessas doenças com prognóstico às vezes muito reservado, dados hemodinâmicos confiáveis se constituem em aspecto diagnóstico fundamental.

A manometria é convencionalmente realizada por meio de cateteres diagnósticos e sistemas de aferição de pressão, baseados em coluna de fluido e em um diafragma oscilométrico, que transmitem o impulso mecânico detectado na ponta do cateter convertido em sinal elétrico, o qual, por sua vez, é transduzido em registro gráfico e com digitação eletrônica. Esse sistema de transdução de sinais físicos é sujeito a ar- tefatos que o exacerbam e/ou atenuam, resultando em valores pressóricos errôneos, pois são inerentemente influenciados e deformados pela resposta dinâmica de frequência do sistema como um todo. Como parte do relativo negligenciamento a que está relegado atualmente o método da manometria durante o cateterismo cardíaco, não se observa, nos laboratórios em geral, o cuidado com a verificação periódica das condições ideais de resposta dinâmica do sistema de transdução das pressões intravasculares.

O método manométrico miniaturizado é um sistema de aferição de pressão intravascular que adapta um sensor de pressão diretamente a um fio-guia de 0,014", semelhante aos fios-guia comumente utilizados em angioplastias coronárias, o qual permite o registro de gráficos pressóricos simultâneos e comparativos entre o dispositivo e o cateter-guia. Desde 1996, a partir da utilização clínica por Pijls e De Bruyne ${ }^{4}$, na avaliação funcional de estenoses coronárias, o método consolidou-se na prática da Cardiologia Intervencionista, em diversificadas aplicações relacionadas com a mensuração da reserva fracionada de fluxo coronário e da reserva vasodilatadora coronária.

O objetivo desta publicação foi descrever a utilização pioneira em nosso meio do sistema pressure-wire na obtenção de curvas pressóricas fidedignas em pacientes com doenças cardíacas estruturais congênitas e adquiridas, quando são necessários registros manométricos confiáveis da circulação pulmonar, gradientes transvalvares e estenoses de condutos cirúrgicos ou vasculares nativos.

\section{MÉTODOS}

\section{Descrição do dispositivo}

A descrição feita neste artigo se refere aos sistemas RADI Analyzer ${ }^{\circledR}$ Xpress e PressureWire $^{\circledR}$ Certus (St. Jude Medical Inc., St. Paul, Minessota, Estados Unidos), utilizados em nosso laboratório de Cardiologia Intervencionista desde 2007. Nas modalidades que os temos utilizado, os dispositivos de manometria são fios-guia 0,014" com ponta do tipo floppy, semelhantes aos utilizados em angioplastias coronárias, com um sensor de pressão localizado na junção entre a extremidade distal mais radiopaca e o corpo do fio, a cerca de 3 $\mathrm{cm}$ da ponta. Os guias são construídos em formato core-to-tip e possuem transmissão do torque numa razão próxima a 1:1. Existe, no mercado nacional, o pressure-wire hidrofílico Certus ${ }^{\circledR}$ (Figura 1).

O pressure-wire é conectado ao console RADI Analyzer $^{\circledR}$ Xpress para transmissão dos dados por meio de um adaptador na porção proximal do guidewire. $\mathrm{O}$ sistema permite a medida de pressões na faixa entre -30 mmHg e $300 \mathrm{mmHg}$, com acurácia de $\pm 1 \mathrm{mmHg}$ mais $\pm 1 \%$ do valor obtido entre $-30 \mathrm{mmHg}$ e $50 \mathrm{mmHg}$; e $\pm 3 \%$ do valor obtido entre $5 \mathrm{mmHg}$ e $300 \mathrm{mmHg}$. A frequência de resposta do sistema é de $25 \mathrm{~Hz}$, o 


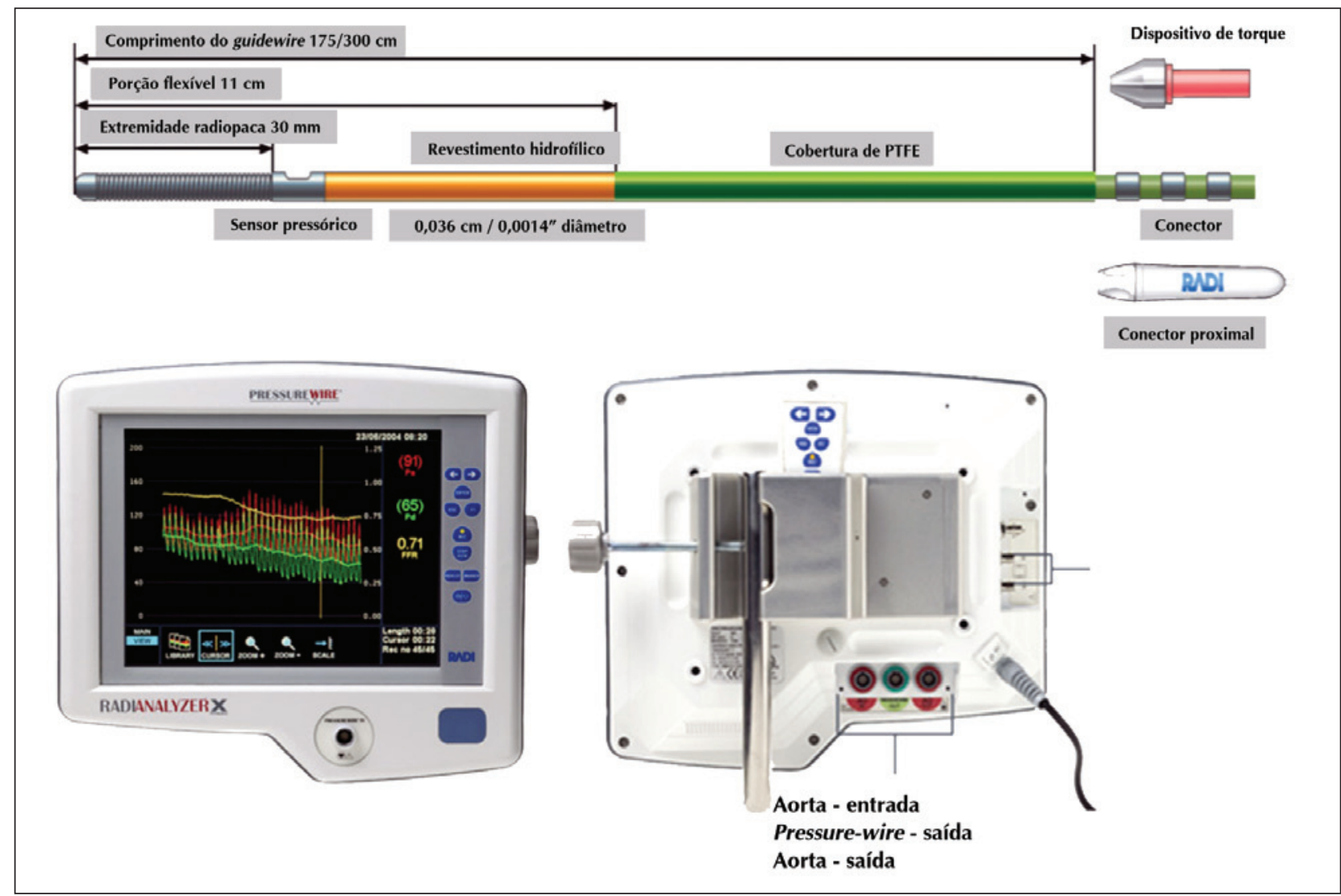

Figura 1. RADI Analyzer ${ }^{\circledast}$ Xpress (St. Jude Medical Inc., St. Paul, Minnesota, Estados Unidos) e pressure-wire ${ }^{\circledast}$.

que permite a obtenção de sinal com grande precisão, mesmo em frequências cardíacas elevadas.

O console RADI Analyzer ${ }^{\circledR}$ possui dimensões de $29 \times 12 \times 31,5 \mathrm{~cm}$, pesando em torno de 4,4 kg (com o transformador) e operando em faixa de voltagem de 100 a 240 volts. Idealmente, deve ser operado em ambientes climatizados, a temperaturas entre 15 e $30^{\circ} \mathrm{C}$ e com umidade ambiente entre 30 e $75 \%$.

\section{Descrição do procedimento}

Pacientes submetidos a ceteterismo cardíaco em que a manometria de câmaras cardíacas ou vasos sistêmicos ou pulmonares se constituía num dado fundamental para a decisão terapêutica, sem a possibilidade de obtenção das informações de maneira habitual mediante o uso de cateteres diagnósticos, foram selecionados para inclusão neste estudo. Eles não foram descritos individualmente, mas agrupados de acordo com a indicação do cateterismo cardíaco e conforme a doença de base.

Os procedimentos foram realizados utilizando-se cateteres diagnósticos convencionais, diferentemente do contexto de avaliação de estenoses coronárias, em que cateteres-guia são escolhidos, em vista da perspectiva terapêutica com angioplastia coronária. Não houve comprometimento da qualidade dos traçados obtidos dessa maneira.

Quando se utiliza o pressure-wire, o console fica interposto entre o sistema convencional transdutor de pressão, montado na mesa de exame, e o polígrafo. Existem quatro conexões no console monitor, sendo uma na face frontal e três no verso, nas quais se conectam, respectivamente, o pressure-wire via adaptador na face frontal; a entrada do sinal da pressão captado pelo cateter diagnóstico e coluna de líquido; a saída do sinal da pressão transmitida pelo cateter (polígrafo $\mathrm{P} 1)$; e a saída do sinal de pressão pelo pressure-wire (polígrafo - P2) na face dorsal. Após checar as conexões, o console é ligado, e o transdutor de pressão convencional é exposto à pressão atmosférica e zerado nesse nível, para o polígrafo e o console. Esse passo é seguido de exposição à pressão atmosférica e da calibração do pressure-wire após sua imersão em filme raso de salina, ainda no sistema de envoltório em que é fornecido. A sequência dos passos é mostrada no monitor do console. Com o dispositivo calibrado para uso, o pressure-wire é inserido no cateter, via conector em $\mathrm{Y}$, e posicionado de forma que o sensor de pressão se situe na extremidade distal do cateter, para equalização de pressões. A equalização de pressões deve ser realizada após remover a agulha introduto- 
ra (passador) do conector em Y, e fechar-se a valva deste suavemente, sem remanescentes de contraste ou sangue no cateter. Qualquer diferença mínima de pressão entre o cateter e o pressure-wire é removida eletronicamente (equalização). Após a realização das medidas pressóricas, ao retirar o pressure-wire, nova checagem da equalização é feita, para se certificar da qualidade dos dados obtidos.

As oscilações das pressões obtidas de acordo com o ciclo respiratório são geralmente minimizadas solicitando-se ao paciente uma pausa expiratória ou ao anestesista, quando o procedimento é realizado sob sedação e suporte respiratório invasivo, como ocorrido no presente estudo.

\section{RESULTADOS}

\section{Pacientes com atresia pulmonar com colaterais sistêmico-pulmonares}

$\mathrm{Na}$ atresia pulmonar com comunicação interventricular (APCIV), o fluxo pulmonar é mantido através do canal arterial, que se mantém patente, ou através de colaterais sistêmico-pulmonares. Para o planejamento cirúrgico, a documentação dos vasos colaterais e das respectivas pressões intravasculares é de fundamental importância, uma vez que a presença de hiperfluxo por vasos colaterais não estenóticos, ou de segmentos pulmonares com dupla irrigação por colaterais e ramos da artéria pulmonar, pode ocasionar regimes pressóricos elevados e contraindicar ou predizer resultados adversos, de acordo com a estratégia cirúrgica adotada. ${ }^{5} \mathrm{~A}$ documentação exata das pressões intravasculares nesses trajetos é importante, tanto nos pacientes em que o planejamento cirúrgico é a correção univentricular, quanto naqueles em que é biventricular.

Os vasos colaterais sistêmico-pulmonares frequentemente são tortuosos e de pequeno calibre, com estenoses por vezes angiograficamente não demonstráveis, devido à sobreposição vascular e à prática rotineira da angiografia monoplanar. Nessas circunstâncias, o método do pressure-wire permite documentar vasos colaterais com estenoses proximais protetoras, assim como a ausência delas, de maneira bastante confiável, reduzindo, dessa forma, os riscos associados à cateterização seletiva desses vasos. A manipulação do guidewire pressórico não apresenta dificuldades, uma vez que o cateter diagnóstico (JR 5 F, AL1 5 F ou MP 5 F) encontra-se próximo à origem do vaso colateral. Além disso, sua manobrabilidade é excelente, aproximando-se da que habitualmente se verifica durante procedimentos intracoronários.

A utilização dessa técnica apresenta um aspecto favorável adicional, que reside no fato de não se comprometer a única fonte de fluxo sanguíneo pulmonar em alguns desses doentes. A Figura 2 ilustra um caso de APCIV tipo C, de acordo com a classificação de Barbero-Marcial, ${ }^{6}$ em que foram cateterizados todos os
Uso do Pressure-Wire na Avaliação das Cardiopatias Estruturais

vasos colaterais e documentadas as pressões intravasculares correspondentes.

\section{Cateterismo cardíaco pré-cirurgia de Glenn}

Após a descrição original da cirurgia de Glenn, sua aplicação clínica, de maneira a anastomosar a veia cava superior terminolateralmente à artéria pulmonar, foi feita por Azzolina et al.,7 em 1972. Na avaliação pré-operatória do shunt cavo-pulmonar estagiado, é fundamental a documentação de pressões intravasculares pulmonares $<15 \mathrm{mmHg}$. Frequentemente, no cenário de hipoplasias dos ramos pulmonares, o paciente inicialmente é tratado com um shunt sistêmico-pulmonar do tipo Blalock-Taussig modificado e, por vezes, a cateterização seletiva desses enxertos é laboriosa e não isenta de risco. Ainda quando possível, dificuldades técnicas não permitem a acurada aferição das pressões por cateteres convencionais. O método do pressure-wire, nesse cenário, permite que curvas sejam obtidas de forma segura, sem se comprometer o fluxo por esses shunts, para se obterem valores pressóricos adequados. Outro aspecto significante é que, na presença de anomalias da arborização pulmonar, em consequência do shunt criado, a presença do guidewire não interfere na morfologia da curva pressórica e é manobrável a longas distâncias, ao contrário do cateter diagnóstico, que, em geral, não tem dirigibilidade apropriada para alcançar os sítios de obstrução. Na Figura 3, há a documentação de uma caso de cateterismo pré-Glenn em paciente de 8 meses com shunt de Blalock-Taussig modificado, diagnóstico de APCIV, em programação de correção univentricular.

No intraoperatório, quando existe fluxo pulmonar transvalvar, geralmente este é mantido, salvo quando, depois de realizada a anastomose cirúrgica, a pressão pulmonar se encontra elevada. É possível, no intraoperatório, utilizar a medida do pressure-wire para decidir pela perviedade da valva pulmonar, embora em nosso serviço isso ainda não tenha sido realizado.

\section{Cateterismo cardíaco pré-cirurgia de Fontan}

A implementação desse shunt cavo-pulmonar total deve obedecer a pré-requisitos considerados essenciais para o sucesso a longo prazo. Dentre eles, a documentação de pressão pulmonar média $<15 \mathrm{mmHg}$ e a resistência arteriolar pulmonar $<4 \mathrm{UW} / \mathrm{m}^{2}$ são fundamentais. Pacientes com doenças cardíacas que requerem tratamento do tipo cirurgia de Fontan podem não tolerar nem mesmo pequenos graus de estenose pulmonar. ${ }^{8}$ Habitualmente, a cateterização pulmonar, por um shunt de Glenn, não apresenta dificuldades, porém sistemas mais acurados e isentos de artefatos, que interfiram em curvas pressóricas, possibilitam a documentação de pequenos gradientes anatômicos, que podem passar despercebidos no cenário de regimes pressóricos pouco elevados. Daí o emprego justificado do método do pressure-wire em tais circunstâncias. A Figura 4 mostra um paciente em programação de shunt 

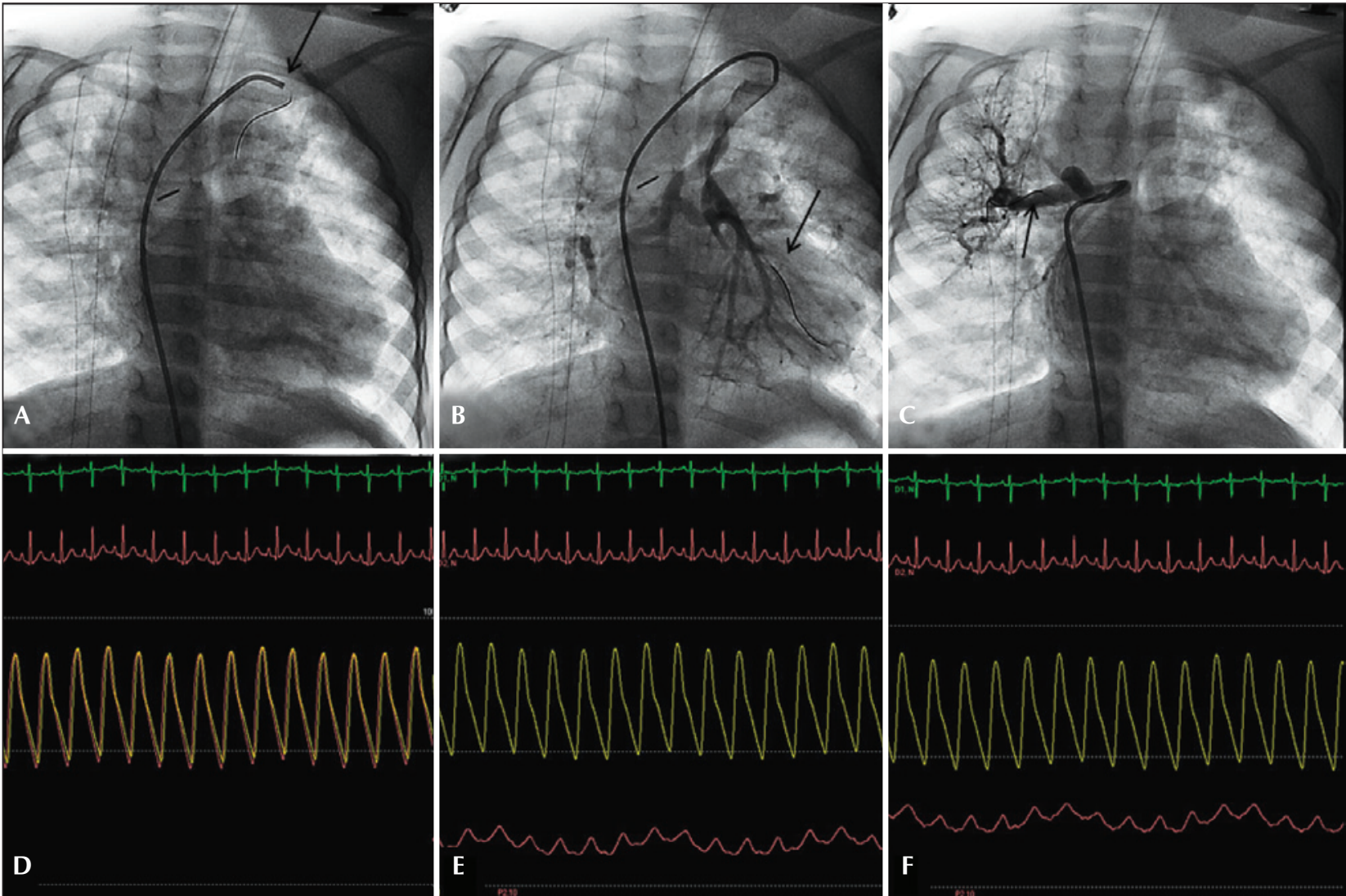

Figura 2. Atresia pulmonar com comunicação interventricular do tipo C (Barbero-Marcial). (A) Cateterização seletiva de colateral bifurcado originando-se na subclávia esquerda, com pressure-wire em posição de equalização de pressões. (B) Pressure-wire no ramo esquerdo da artéria pulmonar, através do sub-ramo mais estenótico do vaso colateral. (C) Colateral para o pulmão direito, lobo superior. (D) Equalização de pressões. (E) Pressão intravascular no ramo esquerdo da artéria pulmonar, lobo inferior, com média de $18 \mathrm{mmHg}$. (F) Pressão intravascular em ramo para o lobo superior do pulmão direito, com média de $21 \mathrm{mmHg}$.

cavo-pulmonar total e diagnóstico de atresia pulmonar, com colateral sistêmico-pulmonar para o pulmão direito, para avaliar conexões interlobares intraparenquimatosas e continuidade das artérias lobares com a artéria pulmonar principal. A presença de arterialização na curva de pressão pulmonar, com gradiente pressórico de $4 \mathrm{mmHg}$ entre o ramo esquerdo (perfundido preferencialmente pelo shunt de Glenn) e o ramo direito da artéria pulmonar (perfundido pelo colateral aortopulmonar), angiograficamente sugere défice perfusional segmentar no pulmão direito.

\section{Cateterismo cardíaco em presença de estenoses de ramos pulmonares}

As anomalias da arborização pulmonar são frequentes com as malformações conotruncais, sobretudo nas variantes da tetralogia de Fallot e, em especial, na atresia pulmonar. Essas estenoses podem ser congênitas ou consequentes a procedimentos cirúrgicos do tipo Blalock-Taussig ou para unifocalização de ramos (Rastelli), por fibrose ou torção no sítio da anastomose. Por vezes, essas estenoses, na ausência de hipertensão pulmonar significativa, são de difícil avaliação executando-se apenas o procedimento manométrico convencional, com pullback do cateter. Nesse cenário, as medidas simultâneas da pressão distal à estenose, com o pressure-wire, e no tronco pulmonar, com o cateter, permitem identificar pequenos gradientes e também estudar vasos de difícil abordagem com o método convencional.

O caso clínico da Figura 5 mostra um paciente com 4 anos e diagnóstico de atresia pulmonar com defeito do septo atrioventricular (AV) não balanceado, submetido a cirurgia de Blalock-Taussig convencional com 1 ano de vida e outro shunt de Blalock-Taussig modificado com 3 anos, após suspeita de estenose do primeiro shunt por avaliação não invasiva, sem confirmação por angiocardiografia. Documentou-se, pelo método do pressure-wire, estenose do ramo esquerdo, que foi tratada com stent e oclusão do shunt central.

\section{Cateterismo cardíaco no pós-operatório de cirurgia com implante de tubo ventrículo direito-tronco pulmonar}

A cirurgia de reconstrução da via de saída do VD habitualmente se faz pela interposição de tubos ven- 
trículo direito-tronco pulmonar (VD-TP), valvulados ou não, que frequentemente se calcificam no pós-operatório tardio, evoluindo com estenoses adquiridas. Geralmente, a medida de pressão sistólica no VD e distalmente ao tubo é suficiente para o diagnóstico, porém, quando estenoses dos ramos estão presentes, a documentação
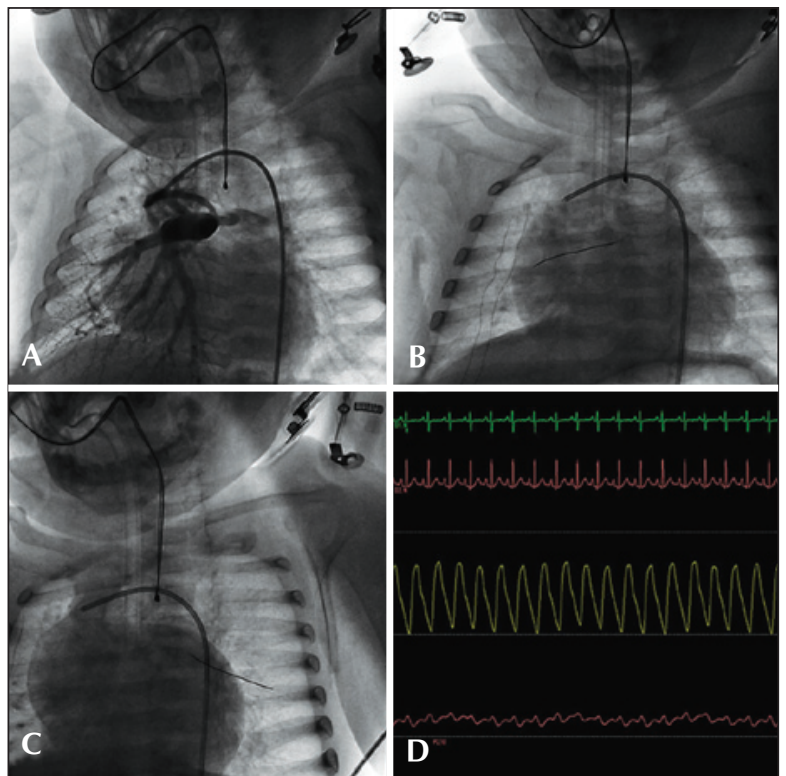

Figura 3. Paciente com atresia pulmonar com comunicação interventricular submetido a shunt de Blalock-Taussig modificado. (A) Arteriografia pulmonar com injeção por shunt de Blalock-Taussig. (B) Pressure-wire posicionado através do tubo de GORE-TEX ${ }^{\circledR}$ no ramo direito da artéria pulmonar. (C) Pressure-wire no ramo esquerdo da artéria pulmonar. (D) Curvas pressóricas aórtica e pulmonar simultâneas, com pressão intravascular pulmonar média em $11 \mathrm{mmHg}$ em ambos os ramos. do nível da obstrução e a significância de cada uma delas é, em grande parte, facilitada pela utilização do pressure-wire. Outro aspecto relevante é que a localização dos gradientes nas anastomoses proximais, distais ou na topografia valvar, às vezes, é dificultada por movimentos bruscos do cateter, em consequência do turbilhonamento do fluxo nessas áreas. O pressurewire também torna mais fácil e confiável a realização do procedimento nessas condições.

\section{DISCUSSÃO}

O sistema manométrico, que emprega o pressurewire para avaliação funcional de estenoses coronárias, foi padronizado, em essência, para determinação da reserva fracionada de fluxo. Nesse sentido, o método tem ganhado aceitação progressiva por sua grande praticidade em situações as mais variadas, desde lesões em tronco coronário esquerdo, estenoses focais seriadas, em bifurcações, e mesmo na presença de afilamentos difusos das artérias coronárias. ${ }^{9}$

No presente trabalho, estendemos o conceito da praticalidade do método manométrico baseado no uso de pressure-wire para diversas aplicações em pacientes portadores de cardiopatias congênitas e adquiridas. As aplicações práticas decorrentes desse conceito são, portanto, expandidas a partir da utilização, em nosso laboratório de Hemodinâmica e Cardiologia Intervencionista, para o contexto específico da atresia pulmonar, como descrito recentemente por Haddad et al., ${ }^{10}$ ao demonstrar a factibilidade e a segurança da técnica em dez pacientes pediátricos.

As curvas pressóricas obtidas com cateteres diagnósticos frequentemente apresentam artefatos provocando reverberação ou atenuação como ringing, overshooting e
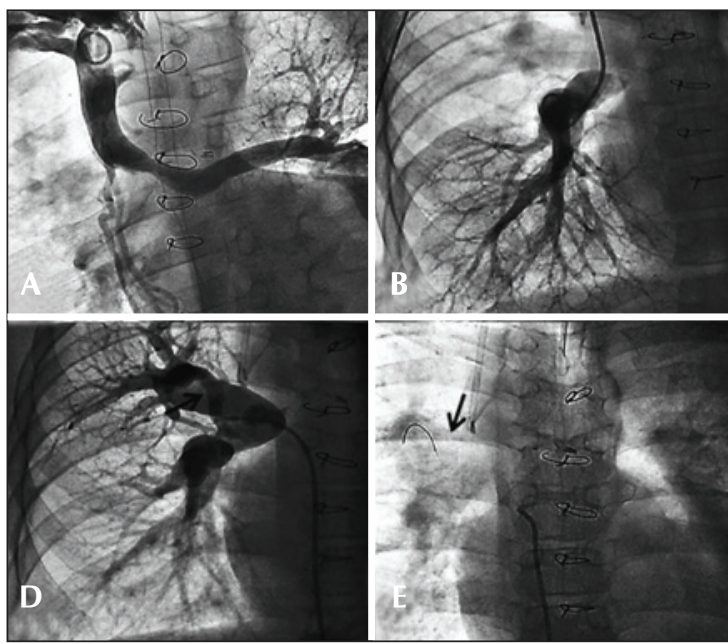
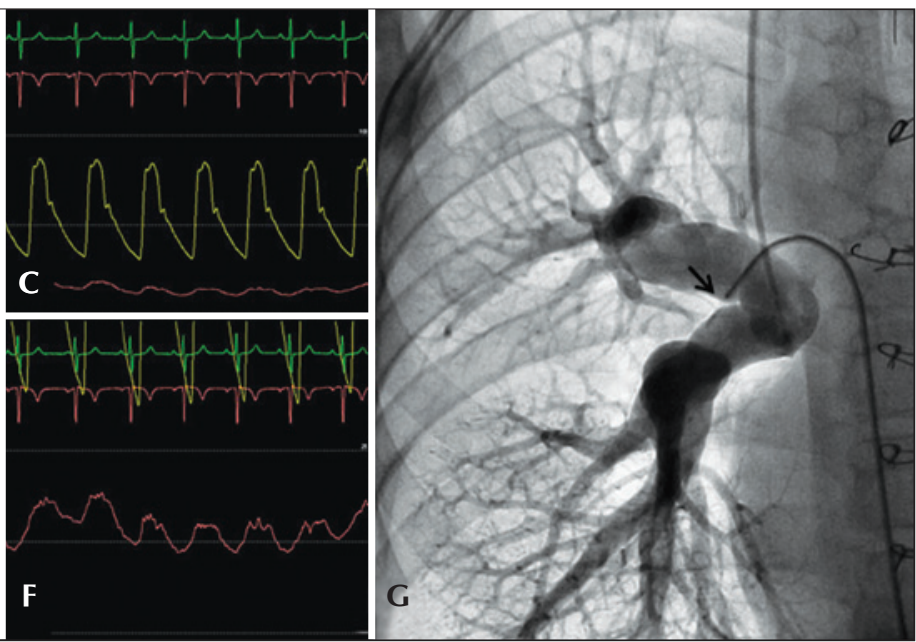

Figura 4. Cateterismo pré-Fontan. (A) Anastomose de Glenn bidirecional com fluxo preferencial para o pulmão esquerdo. (B) Angiografia seletiva do ramo direito da artéria pulmonar através do conduto na cirurgia de Glenn. (C) Pressões aórtica e no ramo esquerdo da artéria pulmonar, em escala de 100 (ramo esquerdo da artéria pulmonar de $10 \mathrm{mmHg}$ ). (D) Colateral aortopulmonar para o lobo superior direito. (E) Pressure-wire no vaso colateral. (F) Pressão intravascular no ramo direito da artéria pulmonar obtida com o pressure-wire (média de $14 \mathrm{mmHg}$ ) em escala de $25 \mathrm{mmHg}$. (G) Angiografia com cateter de Berman ocluindo o vaso colateral, mostrando a irrigação de todo pulmão direito pela artéria pulmonar. 

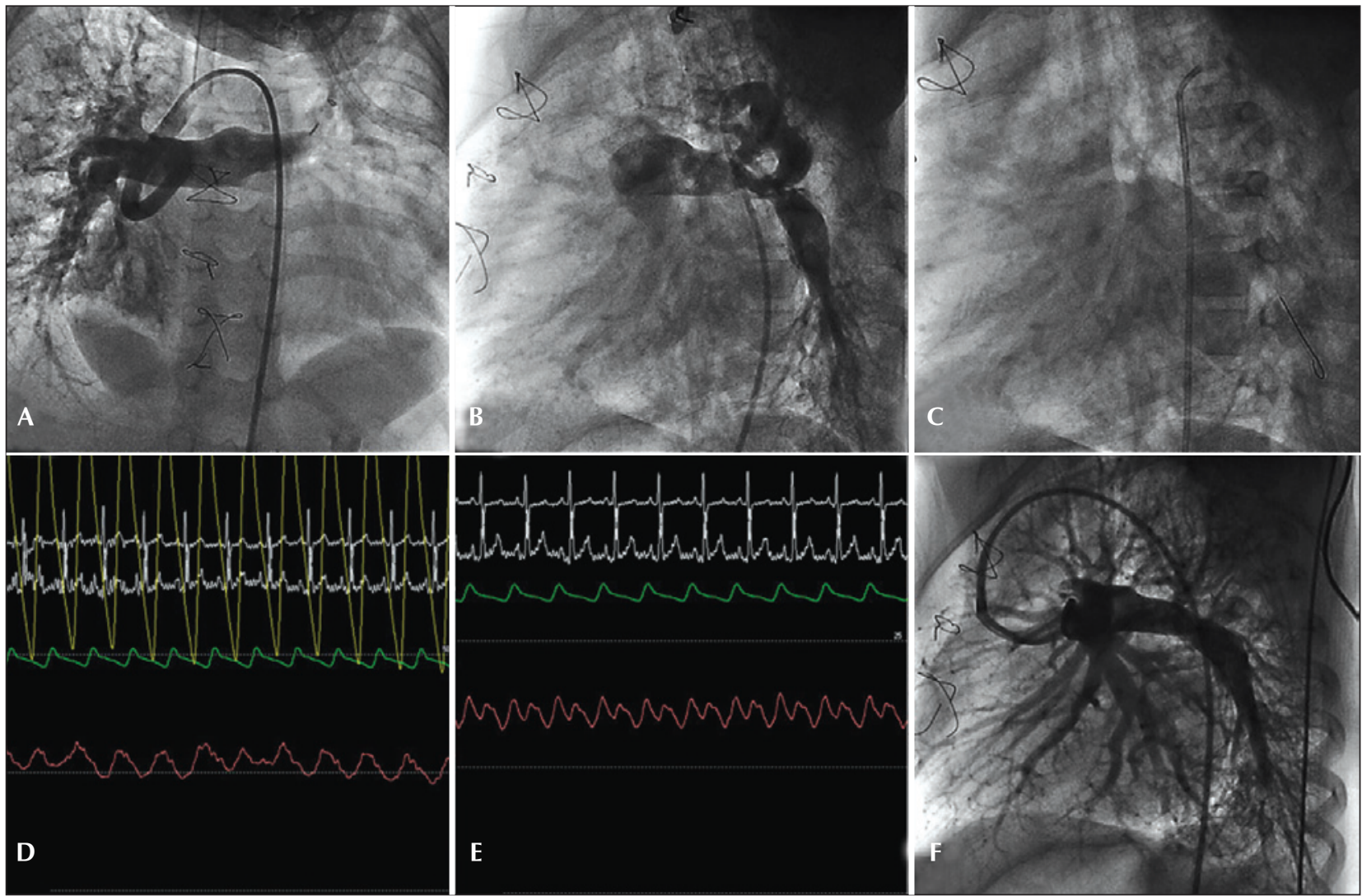

Figura 5. Paciente com dois shunts centrais, com hiperfluxo pulmonar e estenose do ramo esquerdo. (A) Shunt de Blalock-Taussig modificado. (B) Shunt de Blalock-Taussig convencional. (C) Pressure-wire no ramo esquerdo da artéria pulmonar. (D) Manometria do ramo direito da artéria pulmonar com cateter MP $5 \mathrm{~F}$ (pressão média de $25 \mathrm{mmHg}$ em escala de $50 \mathrm{mmHg}$ ). (E) Manometria do ramo esquerdo da artéria pulmonar distal (pressão média de $17 \mathrm{mmHg}$ em escala de $25 \mathrm{mmHg}$ ). (F) Implante de stent no ramo esquerdo da artéria pulmonar com oclusão do shunt de Blalock-Taussig.

damping, que limitam a aquisição de valores pressóricos exatos. Nessas circunstâncias, torna-se impraticável o diagnóstico de gradientes venosos significativos, com o agravante de que, nessa parte do sistema circulatório, mesmo pequenos gradientes podem ter muita relevância clínica. O sistema manométrico baseado no uso de pressure-wire, por se tratar de um dispositivo que afere pressão sem necessidade de diafragmas e colunas interpostas de líquido, permite que valores pressóricos sejam registrados de maneira mais acurada e sem artefatos, nos diversificados contextos aqui abordados. O sistema manométrico com o pressure-wire, em nosso laboratório de Hemodinâmica e Cardiologia Intervencionista, tem permitido a obtenção de pressões intravasculares pulmonares e em condutos vasculares nativos ou decorrentes de intervenções cirúrgicas, de maneira segura e com menor risco em comparação com a técnica habitualmente utilizada, como exemplificado nos casos descritos.

Um outro aspecto de potencial muito relevante será a utilização do pressure-wire como ferramenta para cálculo do fluxo através de um conduto. As versões mais novas do PressureWire ${ }^{\circledR}$ Certus e do console RADI Analyzer ${ }^{\circledR}$ Xpress permitem a medida do fluxo e a estimativa de débito por método baseado em sistema termodiluidor. Em paciente com cardiopatias que cursam com hiperfluxo pulmonar frequentemente não é possível aplicar o princípio de Fick para sua quantificação adequada. Em adição, eventualmente, casos com atresia pulmonar e colaterais sistêmico-pulmonares e/ou canal arterial patente são rotulados, superficialmente, como portadores de hipofluxo pulmonar. Entretanto, em tese, pode se traduzir no contrário, mediante a cateterização seletiva de todos os vasos colaterais e a medida da somatória do fluxos através destes, uma vez que o resultado pode vir a ser de verdadeiro hiperfluxo pulmonar. Isso permitiria também a estimativa de resistências pulmonares individualmente de todos os segmentos pulmonares assim perfundidos. Convém enfatizar a natureza especulativa dessa condição, necessitando de dados ainda não disponíveis na literatura para tal documentação.

\section{CONCLUSÕES}

Em nossa prática recente de cateterismo cardíaco diagnóstico de várias doenças congênitas e adquiridas, o método manométrico miniaturizado usando o pressure-wire tem se tornado virtualmente indispensável 
em diversos contextos. Trata-se de método diagnóstico complementar importante, não apenas nos casos em que existe dificuldade na utilização dos meios habituais para aquisição de pressões intravasculares, mas também nos casos rotineiros, uma vez que permite a obtenção de traçados com menos artefatos. O registro de curvas pressóricas simultâneas, sem a necessidade de duas punções vasculares ou de cateteres mais calibrosos, permite graduar de maneira muito satisfatória estenoses com significativa repercussão hemodinâmica, em diversos contextos clínicos e pós-cirúrgicos. É previsível também a expansão futura de seu uso, com outras finalidades diagnósticas complementares.

\section{CONFLITO DE INTERESSES}

Não há.

\section{FONTE DE FINANCIAMENTO}

Não há.

\section{REFERÊNCIAS}

1. Buzzi A. Claude Bernard on cardiac catheterization. Am J Cardiol. 1959;4:405.

2. Mullins CE. Cardiac catheterization in congenital heart disease: pediatric and adult. Massachusetts: Blackwell; 2006
3. Sibley DH, Millar HD, Hartley CJ, Whitlow PL. Subselective measurement of coronary blood flow velocity using a steerable Doppler catheter. J Am Coll Cardiol. 1986;8(6):1332-40.

4. Pijls NH, De Bruyne B, Peels K, Van Der Voort PH, Bonnier HJ, Bartunek J, et al. Measurement of fractional flow reserve to assess the functional severity of coronary artery stenosis. N Engl J Med. 1996;334(26):1703-8.

5. Gosse-Wortmann L, Yoo SL, Van Arsdell G, Chetan D, Macdonald $C$, Benson $L$, et al. Preoperative total pulmonary blood flow predicts right ventricular pressure in patients early after complete repair of tetralogy of Fallot and pulmonary atresia with major aortopulmonary collateral arteries. J Thorac Cardiovasc Surg. 2013;146(6):1185-90.

6. Croti UA, Barbero-Marcial M, Jatene MB, Riso AA, Tanamati C, Aiello VD, et al. Classificação anatômica e correção cirúrgica da atresia pulmonar com comunicação interventricular. Rev Bras Cir Cardiovasc. 2001;16(4):321-36.

7. Azzolina G, Eufrate S, Pensa P. Tricuspid atresia: experience in surgical management with a modified cavopulmonary anastomosis. Thorax. 1972;27(1):111-5.

8. Sievert H, Qureshi SA, Wilson N, Ziyad M Hijazi ZM, editors. Percutaneous interventions for congenital heart disease. London: Informa Healthcare; 2007.

9. Pijls NH, Sels JW. Functional measurement of coronary stenosis. J Am Coll Cardiol. 2012;59(12):1045-57.

10. Haddad JL, Novaes GC, Figueiredo GL, Lemos DC, Macedo LG, Lago IM, et al. Use of the pressure wire method for measuring pulmonar arterial pressures in patients with pulmonar atresia. Cardiol Young. 2014 Jan 15:1-8. [Epub ahead of print] 